\title{
Eccentric Sequence of Graphs
}

\author{
S. Meenakshi, Deepika .K, R. Abdul Saleem
}

\begin{abstract}
The distance d $(u, v)$ from a vertex $u$ of graph $G$ to a vertex $v$ is the length of a shortest $u$ to $v$ path. The eccentric sequences were the first distance related sequences introduced for undirected graphs. The eccentricity $e(v)$ of $v$ is the distance of a farthest vertex from $v$. The eccentric sequence of a graph $G$ is a list of the eccentricities of vertices of graph $G$ arranged in non-decreasing order. In this paper we determine the eccentric sequence of join of an empty graph and path graph(ie fan graph) and the eccentric sequence of the Cartesian product of paths $P 2$ and Pn (ie Ladder graph).
\end{abstract}

Keywords : Cartesian product of graphs, Degree sequence of graphs, Eccentric Sequence of graphs, Join of a graph.

\section{INTRODUCTION}

In graph theory, representing a sequence as a list of arithmetical values or numbers is more preferable than an independent number with respect to the graph. It is convenient to use sequences when compared to using a single number or an invariant as the sequence carries more information about the graph than an invariant. Degree Sequence, Eccentric Sequence, Distance Degree Sequence, Status Sequence, Path Degree Sequence are some sequences representing a graph.

The first sequence to be introduced was the Degree Sequence. Havel and Hakimi independently worked on how a Degree Sequence realizes a graph. Distance related sequences namely Eccentric Sequences were introduced for undirected graphs. Lesniak, Ostrand, Behzad and Simpson and Nandakumar made a major contribution in the concept of Eccentric Sequences. Later Randiac made a major contribution in the distance based sequences namely the path degree sequence and distance degree sequence. These sequences were initially used extensively in the study of differentiating molecules in chemistry by modeling a chemical form with its molecular graph. Embedding trees in lattice graphs using distance degree sequences were developed by Kennedy and Quintas. Bloom et.al later continued to study the distance degree sequence.

In this paper we have discussed the eccentric sequence of join graph and the Cartesian product of graphs.

Revised Manuscript Received on December 5, 2019.

* Correspondence Author

S. Meenakshi*, Associate Professor, Dept of Mathematics, VISTAS, Chennai, Tamil Nadu

Email: meenakshikarthikeyan@yahoo.co.in

Deepika K., Research Scholar, Dept of Mathematics, VISTAS, Chennai, Tamil Nadu

Email: maths1988@gmail.com

R. Abdul Saleem, Dept of Mathematics, The Quaide Milleth College for Men, Chennai, University of Madras

\section{PRELIMINARIES}

Let $\mathrm{G}(\mathrm{V}, \mathrm{E})$ to be a graph where $\mathrm{V}$ is the list of vertices and $\mathrm{E}$ is the list of edges. Here $\mathrm{G}$ is a finite undirected graph without multiple edges and self loops.

Consider a, b as two vertices in Graph G. The length of shortest $a-b$ path from the vertex a to the vertex $b$ is called the distance $d(a, b)$ from a to $b$.

The list of number of incident edges of all vertices of the graph is called the Degree Sequence of a graph.

The distance of a farthest vertex from $g$ is called the eccentricity e(g) of vertex $g$.

Diameter of the graph $G$ is the highest of the eccentricities of G. Radius of graph $G$ is the least of the eccentricities of $\mathrm{G}$.

If all the vertices of the graph $G$ have same eccentricity, then the graph is said to be self- centered.

A vertex $\mathrm{x}$ is an eccentric vertex of another vertex $\mathrm{y}$ if $\mathrm{d}(\mathrm{x}, \mathrm{y})=\mathrm{e}(\mathrm{x}), x \neq y$

The Eccentric Sequence of a connected graph is a list of the eccentricities of its vertices.

Example:

G

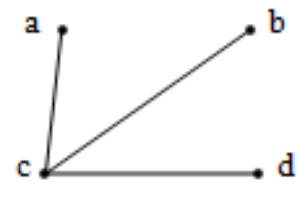

Fig (i)

The Eccentricity Sequence of the above graph G is 1,2,2,2.

\section{THE ECCENTRIC SEQUENCE OF JOIN GRAPH (FAN GRAPH) AND THE ECCENTRIC SEQUENCE OF CARTESIAN PRODUCT OF PATHS $P_{2}$ AND $P_{n}($ LADDER GRAPH)}

\section{A. Definitions}

Let $\mathrm{H}_{1}$ and $\mathrm{H}_{2}$ be two graphs with disjoint vertex sets $\mathrm{V}_{1}$ and $\mathrm{V}_{2}$ and edge set $\mathrm{X}_{1}$ and $\mathrm{X}_{2}$. The union $\mathrm{H}_{1} \mathrm{U} \mathrm{H}_{2}$ along with every edge joining the vertices of $\mathrm{V}_{1}$ and $\mathrm{V}_{2}$ is defined as the Join $\mathrm{H}=\mathrm{H}_{1}+\mathrm{H}_{2}$ graphs $\mathrm{H}_{1}$ and $\mathrm{H}_{2}$.

The Cartesian product $\mathrm{G} \square \mathrm{H}$ of graphs $\mathrm{G}$ and $\mathrm{H}$ is a graph such that the vertex set of $\mathrm{G} \square \mathrm{H}$ is the Cartesian product $\mathrm{V}(\mathrm{G}) \times \mathrm{V}(\mathrm{H})$; and two vertices $\left(\mathrm{a}, \mathrm{a}^{\prime}\right.$ ) and (b, b') are adjacent in $\mathrm{G} \square \mathrm{H}$ if and only if either $\mathrm{a}=\mathrm{b}$ and $\mathrm{a}^{\prime}$ is adjacent to $b^{\prime}$ in $H$, or $a^{\prime}=b^{\prime}$ and $a$ is adjacent to $b$ in $G$.

Let us consider $\overline{K_{n}}$ as the empty graph on n nodes and $P_{m}$ as the path graph on m nodes. A Fan Graph $F_{n, m}$ is the graph join $\overline{K_{n}}+\mathrm{P}_{\mathrm{m}}$. 


\section{Eccentric Sequence of graphs}

We determine the Eccentric sequence of Fan Graph $\mathrm{F}_{\mathrm{n}, 2}$. It is the graph join of an empty graph $\overline{K_{n}}$ and path graph $\mathrm{P}_{2}$.

\section{B. Theorem 1}

The Eccentric Sequence of the Fan graph $F_{n, 2}$ is given by $1,1,2,2, \ldots 2$.

Proof:

We consider the fan graph $F_{1,2}$ with vertices a, b and $\mathrm{v}_{1}$

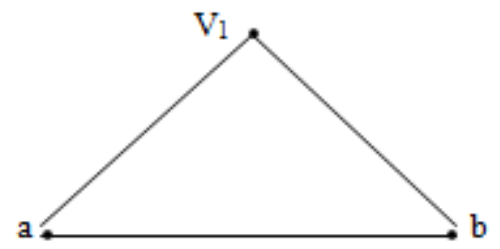

Fig (ii) Graph $F_{1,2}$

The eccentricities of the vertices $a, b$ and $v_{1}$ are $1,1,1$ in that order. So the Eccentric Sequence of the above said graph is $1,1,1$.

We now consider the fan graph $\mathrm{F}_{\mathrm{n}, 2}$.

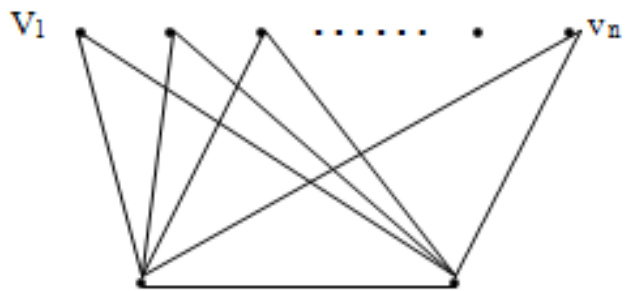

a $\mathrm{b}$

Fig (iii) graph fn,2.

$\overline{K_{n}}$ is a graph with $\mathrm{n}$ nodes. $\mathrm{P}_{2}$ is a path with 2 nodes.

Here $a, b$ are the vertices of the path graph $P_{2}$. The eccentricity of these two vertices is 1 .

So the Eccentricity Sequence is 1,1

Similarly the eccentricity of the vertex $v_{1}$ of the empty graph $\overline{K_{n}}$ is 2 .

Continuing this way, the eccentricities of the other remaining vertices $\mathrm{v}_{2}, \mathrm{v}_{3}, \ldots, \mathrm{v}_{\mathrm{n}}$ of the empty graph $\overline{K_{n}}$ are $2,2,2 \ldots .2$. Therefore we obtain the Eccentric Sequence of the empty graph $\overline{K_{n}}$ and Path graph $\mathrm{P}_{2}$ as

$(1,1,2,2,2, \ldots 2)$.

Hence the theorem.

\section{Theorem 2}

The Eccentric Sequence of the Cartesian Product of paths $P_{2}$ and $\mathrm{P}_{\mathrm{n}}$ ( The Ladder Graph $\mathrm{L}_{\mathrm{n}}$ ) is given by

(i) $\left((n-i)^{2},(n-(i+1))^{4}, \ldots(n-2)^{4},(n-1)^{4}, n^{4}\right)$

when $\mathrm{n}$ is odd.

(ii) $\left((n-i)^{4},(n-(i+1))^{4}, \ldots(n-2)^{4},(n-1)^{4}, n^{4}\right)$

when $n$ is even.

Proof:

The Eccentric Sequence of the Ladder Graph $L_{1}$ and $L_{2}$ is 1,1 and 2,2,2,2 respectively which is trivial.

We prove the result for $n \geq 3$.

When $\mathrm{n}$ is odd

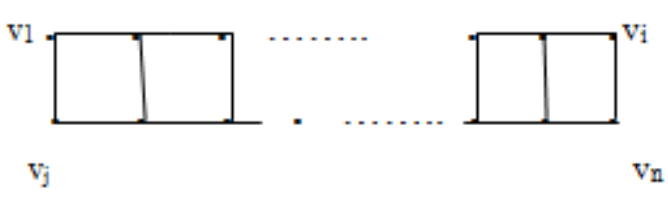

Fig (iv) Graph Ln

The eccentricities of the vertices $\mathrm{v}_{1}, \mathrm{v}_{\mathrm{i}}, \mathrm{v}_{\mathrm{j}}, \mathrm{v}_{\mathrm{n}}$ are $\mathrm{n}$

The eccentricities of the vertices $v_{2}, v i-1, v_{j+1}, v_{n-1}$ are $n-1$. Continuing this way the eccentricities of the two middle vertices are $\mathrm{n}$ - i.Therefore the Eccentric Sequence of the Ladder Graph $L_{n}$ for odd $\mathrm{n}$ is

$$
\left((n-i)^{2}(n-(i+1))^{4}, \ldots(n-2)^{4},(n-1)^{4}, n^{4}\right)
$$

We now prove the result for even $n$

The eccentricities of the vertices are $v_{1}, v_{i}, v_{j}, v_{n} n$

The eccentricities of the vertices $v_{2}, v i-1, v_{j+1}, v_{n-1}$ are $n-1$

The eccentricities of the two $\mathrm{p}$-airs of middle vertices

are

$\mathrm{n}$ - i.Therefore the Eccentric Sequence of the

Ladder Graph $\mathrm{L}_{\mathrm{n}}$ for even $\mathrm{n}$ as

$\left((n-i)^{4},(n-(i+1))^{4}, \ldots(n-2)^{4},(n-1)^{4}, n^{4}\right)$

Hence the theorem

\section{CONCLUSION}

The Eccentric Sequence of the Join Graph (Fan graph) and the Eccentric Sequence of the Cartesian product of paths $P_{2}$ and $P_{n}$ ( The Ladder Graph $L_{n}$ ) have been computed in this paper. Also several properties of eccentric sequence of graphs have been computed by various researchers. Other researchs in this field include the eccentric sequence of self complementary graphs, eccentric sequence in digraphs etc. Eccentric Sequence plays a major role in stratified graphs. The study of eccentric sequence of graphs gives rise to many open problems. To compute the eccentric sequence of some classes of graphs itself is difficult. In future such open problems can be considered and discussed. Also various properties can be deteremined.

\section{REFERENCE}

1. M. Behzad and J.E Simpson, "Eccentric sequences and eccentric sets in graphs,” Discrete Mathematics, Vol. 16(3), pp. 187-193, 1976.

2. F. Buckley, F. Harary, Distance in graphs, Addison-Wesley Publishing 3. Company, 1990 .

4. Chithra M.R., Distance degree graphs in the Cartesian product of graphs, J. Discrete Math. Sci. Cryptogr. 18 (6) (2015), 743-750.

5. P. Erdos and T.Gallai, "Graphs with prescribed degrees of vertices," Matematikai Lapok, Vol. 11pp. 264-274, 1960(Hungarian).

6. W. Imrich, S. Klavzar, R. Hammack, Handbook of Product

7. Graphs, CRC Press (2011).

8. L.Ms.Lesniak-Foster, "Eccentric sequences in graphs," Periodica Mathematica Hungarica, Vol. 6(4), pp. 287-293, 1975.

9. Medha I.H, Distance Degree Regular Graphs and Distance Degree

10. Injective Graphs: An Overview, J. Discrete Math. (2014).

11. R Nandakumar, On some eccentric properties of graphs[PhD Thesis], Indian Institute of Technology, New Delhi, India, 1986.

12. J. Gimbert and N. Lopez, "Eccentric sequences and eccentricity sets in digraphs," Ars Combinatoria, vol. 86, pp. 225-238, 2008.

13. J. Akiyama, K. Ando, and D. Avis, "Eccentric graphs," Discrete Mathematics, vol. 56, no. 1, pp. 1-6, 1985. 


\section{AUTHORS PROFILE}

S. Meenakshi profile which contains their education details, their publications, research work, membership, achievements, with photo that will be maximum 200-400 words.

Deepika K ., Research scholar, VISTAS , Chennai

Publications: The minimum efficient dominating energy of graphs.

R. Abdul Saleem profile which contains their education details, their publications, research work, membership, achievements, with photo that will be maximum 200-400 words. 